\title{
KOMMUNIKATION ALS BASIS DES FSU: SPRACHE, WISSEN, KULTUR
}

\author{
BARbARA SKOWRONEK, Alicja SKOWRONEK
}

\section{Kommunikation als gesellschaftliches Phänomen}

Es gibt keine einheitliche, allumfassende, von allen akzeptierte Definition der Kommunikation (Heath/Bryant 2008: 46), weil Kommunikation ein vielseitiges Phänomen der Informationsübermittlung ist, das unter verschiedenartigen Aspekten untersucht werden kann, entsprechend unterschiedlich sind die Untersuchungsbereiche der Kommunikationswissenschaft. Mit ihren Arbeiten zur Informationstheorie (aus der Technik der Telekommunikation) haben Shannon/ /Weaver 1949, Wiener 1954 einen wesentlichen Beitrag zur Entwicklung der Kommunikationstheorie geleistet. Es wurde versucht, Vorschläge der Schemata der Energietransmission zur Übermittlung elektromagnetischer und elektroakustischer Wellen zwischen den Sende- und Empfangsgeräten zu erarbeiten (dazu gehören auch die misslungenen Versuche von Tesla). Demnach gehören zur Kommunikation alle Prozeduren, dank denen eine Aussage auf jemanden einwirken kann; wichtig dabei sind der technische und der semantische Bereich und die Effektivität der Transmission. Im linearen Modell für Telekommunikation und Radiowellen vorgesehen, wurde der technische Aspekt besonders stark hervorgehoben: wie genau und wie effektiv gegenüber dem Geplanten lassen sich Kommunikationssignale im Kommunikationsgefüge (Sender - Empfänger Kanal - Kode der Übertragung) senden/übertragen.

Das lateinische communicatio bedeutete die Art und Weise, zusammen zu sein, etwas zusammenzustellen, etwas zu erklären, zu deuten; es bezog sich auf gesellschaftliche Relationen. Folglich ist der Sinn der Kommunikation, etwas gemeinsam zu machen, lat. communico bezieht sich auf 'verbinden', 'zusam- 
menfügen', 'etwas mit jemandem teilen', 'an etwas teilhaben', 'etwas allgemein zugänglich machen'; communio bezieht sich auf 'stärken', 'bekräftigen', 'stützen'; communio, -onis, ist 'Teilnahme', 'Beteiligung', 'Mitteilung', 'Unterredung', 'Austausch', 'Verständigung untereinander', 'Erklären', 'Gemeinschaft'; communis, -e, 'gemeinsam', 'allegmein', 'öffentlich', aber auch 'oberflächlich'. Menschliche Kommunikation ist also Vermittlung, zwischenmenschlicher Kontakt, Verbindung, Ausdruck von zwischenmenschlicher Abhängigkeit und gesellschaftlichen Relationen. Diese Relationen können positiv sein (was die Menschen verbindet, vereint, als Ergänzung und Entgegenkommen, Verständnis) oder negativ (was sie unterscheidet oder gar trennt: Konflikte und Destruktion). Das heißt, in der Kommunikation geht es um das Bestimmen gesellschaftlicher Zusammenhänge, der Rangordnung der menschlichen Kontakte. Folglich ist der Mensch ein kommunizierendes Wesen, homo communicans.

Es lässt sich zwischen interpersonaler, zwischenmenschlicher Kommunikation und Kommunikation über Massenmedien bzw. technisch vermittelter Kommunikation unterscheiden; Kommunikation über Internet und virtuelle Kommunikation sind heute nicht mehr wegzudenken (nach Rost-Roth 2010: 155-156). Zu den Elementen des Kommunikationsgefüges gehören: Kommunikationsteilnehmer (Sender, Empfänger), Botschaft (Kommunikat, Information), Kanal, Rückkoppelung (feed back); nicht unwesentlich sind nach DobekOstrowska (2006: 64) der soziale Kontext, und damit auch Störungen. Die Autorin unterscheidet äußere Störungen (z.B. Krach, Sonnenblendung, Hitze), innere Störungen (z.B. Aufregung, Emotionen, Stereotype und Vorurteile), semantische Störungen (ergeben sich aus Mehrdeutigkeiten, also aus inkorrekten oder falsch eingesetzten Bedeutungen). Auch nach DeVito (1970: 201-210) ist Kommunikation Prozess oder Akt der Informationsübertragung vom Sender zum Empfänger durch einen Kanal mit besonderer Berücksichtigung von Störungen.

Im Kommunikationsprozess lassen sich verschiedene Phasen unterscheiden: Senden/Kodieren, Vermitteln/Übertragen, Empfangen/Dekodieren, mit besonderer Bedeutung der Wechselseitigkeit, Intentionalität und Rückmeldung (Rückkoppelung). Kommunikationsprozesse bestehen aus Elementen, die miteinander kausal verbunden sind: Ursache (Aktion) impliziert Wirkung, das Verhalten des einen verursacht Reaktion des anderen; wer fragt/wer spricht, trägt die Verantwortung (Gedanken sind zollfrei). Die zwischenmenschliche Kommunikation ist als zwischenmenschliche Relationen veränderbar, dynamisch, endet nie, Aktion verursacht Reaktion, Ursache verursacht Wirkung. Ziel der Kommunikation ist das Teilen, der Austausch von Informationen, Gedanken, Gefühlen, Wissen. Kommunikation ist auch bewusstes, überlegtes Handeln, Produkt der bewussten Wahl (auch wenn nur manche Handlungen tatsächlich bewusst und intentional ausgeführt werden).

Menschliche Kommunikation beruht auf einem Informationsaustausch zwischen Menschen mit Hilfe von Symbolen, Zeichen und Verhaltensweisen. Kom- 
munizieren ist ein symbolischer Prozess der Semiose: Zeichen sind materiell, sinnlich wahrnehmbar, aber sie können alles Mögliche vertreten; sie werden (aufgrund eines gesellschaftlichen Abkommens gebildet) zu Symbolen, Symptomen, Ritualen, zum gesellschaftlichen Prozess. Kommunikation verläuft immer im gesellschaftlichen Kontext unter mindestens zwei Personen als bewusstes und gezieltes Handeln, als kreativer, unumkehrbarer, unvermeidlicher, entwicklungsartiger und dynamischer Prozess der Interpretation des Sendens und des Bildens neuer Botschaften (Dobek-Ostrowska 2006: 67). Kommunizieren als Austausch von Informationen zieht Änderungen der Umwelt nach sich (was nicht nur typisch menschlich ist). Seitdem homo communicans sprechen gelernt hat, nutzt er das bewusst oder unbewusst aus, aber er sendet stets Signale, die von anderen Kommunikatoren (meistens) verstanden werden.

Kommunikation ist Interaktion, infolge deren Bedeutungen gebildet werden (Garbner 1967), als Prozess des formalen Kodierens symbolischer Bedeutungen, die innerhalb einer Kommunikationsgemeinschaft gelten und verstanden werden. Semiotisch gesehen, ist Kommunikation ein Prozess der Informationsübertragung vom Sender zum Empfänger, hauptsächlich für eine effektive Verständigung; es ist ein gesellschaftlicher (als effektiv intendierter) Verständigungsprozess, in dem Bedeutungen generiert werden. Kommunikation ist einerseits symmetrischer Informationstransport vom Sender zum Empfänger (die vom Sender vermittelte Information wird ideal so vom Empfänger aufgenommen, was eher selten vorkommt); andererseits ist Kommunikation beabsichtigtes Senden und interpretierendes Empfangen von Informationen in Form von unterschiedlichen Zeichensystemen, darunter Sprache (entsprechend den kommunikativen Absichten des Senders und den Aufnahme- und Interpretationsmöglichkeiten des Empfängers). Auch Selbstgespräche sind quasi gesellschaftlich, sie verlaufen zwischen zwei hypothetischen Gesprächsteilnehmern (Skowronek 2008a: 108). Damit die Zeichenfunktion realisiert werden konnte, war es notwendig, zwischen der Form des Kommunikats und dem Inhalt des Kommunikats zu unterscheiden. Es haben sich zwei entgegengesetzte Positionen bezüglich der Kommunikation herausgebildet (vgl. Tomaszkiewicz 2006: 15f.): von der extrem negativen Position (Kommunikation gibt es nicht, denn sie ist reine Illusion, also unmöglich; Beweise dafür sind zahlreiche Lügen, falsche Deutungen, Falschinterpretationen und Missverständnisse, sowohl in privaten als auch in öffentlichen Kontakten), bis zur extrem positiven Position (jede menschliche Tätigkeit ist gesellschaftliche Kommunikation, jedes Wort, jede Geste, ebenso wie Mangel an Wort und Geste, bedeuten Kommunizieren; keine Antwort ist oftmals auch eine Antwort, also Menschen kommunizieren immer, wenn auch ungewollt).

Daraus ist ersichtlich, dass die Auffassung zur menschlichen Kommunikation evoluierte (Lewicka 2007: 42): 
- von einer statischen (eher technizistischen) Position: sowohl dem Sender wie dem Empfänger wurde Symmetrie des Kodierens und Dekodierens von Informationen zugeschrieben, wie es in allopoietischen Systemen, also Maschinen, der Fall ist; sie sind auf bestimmte Art und Weise vorprogrammiert, auf das Erzeugen von Produkten, die anders sind als sie selbst (arbeiten also nicht selbständig); davon ausgehend, entstand das audiolinguale Lernen;

- zur dynamischen (eher menschenbezogenen) Auffassung der Kommunikation: Kodieren und Dekodieren von Informationen sind als Manifestation gesellschaftlicher Relationen, Kontakte, menschlicher Verhaltensweisen aufzufassen, in denen die kommunizierenden Menschen mit Hilfe unterschiedlicher Zeichensysteme, von denen Sprache nur eins von vielen ist, aufeinander einwirken; der Mensch wird als autopoietisches System aufgefasst, welches selbstbildend, selbstorganisierend, selbstreferentiell ist. Dank der Autopoiesie verläuft die menschliche Erkenntnis (darunter auch das Lernen und Lehren) kreativ: auf der Basis des vorhandenen Wissens und der vorhandenen Fertigkeiten bildet das menschliche Gehirn mentale Repräsentationen der Wirklichkeit, auf der Basis des schon vorhandenen Wissens und der Erfahrung des einzelnen Lerners (und nicht als Abbildung-Kopie der uns umgebenden Welt); aus dieser Voraussetzung erwuchs das konstruktivistische Lernen.

Nach der geisteswissenschaftlichen, humanwissenschaftlichen und gesellschaftlichen Orientierung ist Kommunikation mit Handlungen verbunden, deren Ziel die Übermittlung von Informationen, das Bilden, Übertragen und Interpretieren ist (Kohout 2006: 11). Es soll hier um Kommunikation als intendiertes Senden und interpretierendes Empfangen von Informationen im Sinne unterschiedlicher (sprachlicher und nichtsprachlicher) Zeichensysteme gehen (Tomaszkiewicz 2006). Im Kommunikationsprozess wird prinzipiell Verständnis (oder/aber auch bewusstes Missverständnis, Manipulation) angestrebt.

Heute wird auf eine enge Bindung zwischen Gesellschaft und Kommunikation hingewiesen (Fleischer 2007: 174), die hauptsächlich durch den in der Gesellschaft fungierenden Kode (die Sprache) realisiert wird. Die Gesellschaft fungiert dank der Kommunikation, dank der Fähigkeit zum (Mit)Teilen und (Mit)Handeln. Daher ist auf den interaktiven und gesellschaftlichen Charakter der Kommunikation hinzuweisen (lat. munio bedeutet 'ich baue', 'ich mache es sicher', 'ich errichte, renoviere, verschanze, versichere, befestige, verteidige'; munitor, 'Erbauer von Festungen'). Kommunikation liegt jeder Sprach-KulturKommunikations-Gesellschaft zugrunde (Puppel 2007: 79f).

Kennzeichnend für die menschliche Kommunikation als gesellschaftliches Phänomen und gesellschaftlichen Prozess ist Gemeinschaftlichkeit der öffentlichen Sphäre (das Teilhaben an etwas); Kommunikationsteilnehmer treten als 
Sender - Empfänger auf, als Geber - Nehmer; gemeint sind sowohl einzelne Personen, individuelle Mitglieder einer Kultur-Sprach-Kommunikations-Gemeinschaft (Idiolekte und Idiokulturen), als auch ganze Kultur-Sprach-Kommunikations-Gemeinschaften (Polylekte und Polykulturen) (Puppel: 2007: 77f., Bonacchi 2011, 2013, Grucza 2012). Beide Kommunikationsteilnehmer müssen die Möglichkeit haben, am gesellschaftlichen Sprach- und Kulturgebrauch teilnehmen zu können. Eine Sprachgemeinschaft besteht aus einer Gruppe von Sprachbenutzern, also Kommunikationsteilnehmern: Muttersprachlern und Nichtmuttersprachlern, Sprechern und Hörern, Schreibern und Lesern, die am kulturell geprägten Kommunikationsprozess des ständigen Austausches von Angebot und Nachfrage einer oder mehrerer Sprachen teilnehmen/ teilhaben.

Sprach-, Kultur- bzw. Kommunikationsgemeinschaften sind dynamische, vernetzte Gebilde. Die Sprecher bzw. Kommunikationsteilnehmer in wirklichen Kommunikationssituationen gehören je nach dem sprachlich-kulturellen Kontext zu verschiedenen Kommunikationsgemeinschaften (eine wichtige Rolle spielen dabei die Mechanismen der Gruppenidentitätsbildung). Das Gelingen einer kommunikativen Interaktion hängt u.a. davon ab, ob die Kommunikationsteilnehmer bestimmte kommunikative Voraussetzungen sowie die Art und Weise der eingesetzten Sprachmittel teilen (Bonacchi 2011: 16). Nach Fleischer ist der Kommunikationsmechanismus der Produzent des Gesellschaftssystems. Die Kommunikationsziele innerhalb der Gesellschaft sind folgende: die Teilnahme an etwas und die Gruppenzugehörigkeit, Anpassung des Wissensbestandes der einzelnen Kommunikationsteilnehmer in der Gruppe, das in Bewegung Halten verschiedener öffentlicher Institutionen und Organisationen, die Fähigkeit, das Kulturerbe von Generation zu Generation, die Errungenschaften von Wissenschaft und Kultur weiterzugeben (Fleischer 2007: 174). Alle kommunikativen Handlungen einer Gruppe, einer Gemeinschaft unterliegen der öffentlichen Kontrolle, die eine Art Filter bildet (J. Puppel und S. Puppel 2008).

Daraus ist ersichtlich, der Mensch ist sowohl gesellschaftliches Wesen (homo socius) als auch ein kommunizierendes Wesen (homo communicans).

\section{Kommunikation, Sprache und Kultur}

Auch Sprachwissenschaftler versuchten, ein kommunikatives Modell des Sprechakts zu erarbeiteten: die menschliche Kommunikation wurde lange Zeit als symmetrischer Transmissionsprozess des Inhalts und der Form zwischen dem Sender (Quelle der Information-Nachricht) und dem Empfänger (Ziel der Information-Nachricht) aufgefasst (Tomaszkiewicz 2006). Nach Zabrocki (1966a: 3ff.) verläuft die Kommunikation zwischen dem synthetischen Sprachkode des Senders und dem analytischen Sprachkode des Empfängers. Jakobson unter- 
scheidet die expressive, impressive Funktion, Darstellungsfunktion, metasprachliche, phatische und poetische Sprachfunktion im Kommunikationsakt (1970: 350ff.). In den linearen Modellen der sprachlichen Kommunikation von Jakobson und Zabrocki gab es den Kode, den Kanal, das Kodieren und Dekodieren. Dabei ging es nur (hauptsächlich) um den sprachlichen Kode.

Auf sprachliche Kommunikation bezogen ist auch die Reflexivität in Form von wechselseitigen Erwartungen der Kommunikationsteilnehmer bedeutsam: In der Kommunikation wird intendiert und erwartet. Die Formel von Lasswell (1948) "Wer sagt was mit welchen Mitteln zu wem mit welcher Wirkung?" hat bis heute ihre besondere Bedeutung nicht verloren. Das Axiom von Watzlawick/Beavin/Jackson (1967/2007): „Man kann nicht nicht kommunizieren“, impliziert, dass jede Art des menschlichen Verhaltens Informationen und Beziehungsaspekte beinhaltet, also der Mensch kommuniziert immer; es ist dem Menschen unmöglich, nicht zu kommunizieren: jede menschliche Handlung und sein Verhalten, jede Geste, jedes Wort oder auch Mangel an Geste oder Wort teilen seiner Umgebung etwas mit. Auch das Nichtgesagte kann Sinn haben. Alles ist Kommunikation: wer lebt, kommuniziert (Fleischer 2007: 174). Nach Tomaszkiewicz (2006: 13) ist Kommunikation die Grundlage aller gesellschaftlichen Relationen; Kommunikation bedeutet etwas zusammen stellen, Austausch, Gespräch, jmdm. etwas erklären, zusammen sein. Sprachliche Kommunikation ist in menschlichen Kontakten nicht wegzudenken, sie beruht auf Senden und Empfangen von sprachlichen und nichtsprachlichen Zeichen, wobei das Empfangen interpretativ ist: der Empfänger schreibt den Zeichen (als Informationsträgern) Bedeutung zu (vgl. Tomaszkiewicz 2006: 151).

In diesem Sinne ist die menschliche Kommunikation ein intersemiotisches System (Skowronek 2008a, 2008b): gesellschaftlich effektive Kommunikation impliziert nicht so sehr symmetrischen Informationstransport zwischen Sender und Empfänger, sondern vielmehr interpretierende Aufnahme der vom Sender intendierten Informationen durch den Empfänger: Inhalte, Wissen, Überzeugungen, Gedanken und Vorhaben dienen der interpretierenden Realisierung kommunikativer Absichten der Kommunikationsteilnehmer. Gesellschaftlich effektive Kommunikation impliziert die Verwendung unterschiedlicher Zeichensysteme: sprachlicher (im Hörverstehen, Leseverstehen, Sprechen, Schreiben) und nichtsprachlicher (z.B. als Bilder, Tabellen, Piktogramme, Musik und Bewegung, Licht und Schatten, Ton und Stille, Vordergrund und Hintergrund, Farben, Formen, Prosodie, Körpersprache usw.), die sich vervollständigen und gegenseitig ergänzen. Dies sollte als glottodidaktisch relevant aufgefasst werden. Der kommunizierende Mensch handelt aktiv: gesellschaftlich erwünscht oder unerwünscht; er verwendet dabei haupstächlich die audio-vokale Modalität und/oder die Seh-Tast-Modalität oder beides, also ist Kommunikation ein multimodales Zeichensystem. 
Kommunizieren ist in die kulturelle und technische Entwicklung der Menschheit inkorporiert. Die Rolle der Kommunikation für die Entwicklung der Kultur ist enorm, fundamental. Nach Pisarek (2008: 87) sind Rede (das Sprechen), Schrift und Druck die drei Symbole für Veränderungen innerhalb der zwischenmenschlichen Kommunikation; eigenartig ist, dass diese Veränderungen sich nicht revolutionär vollzogen haben, nicht durch Vernichtung des Alten, sondern fließend, langsam, entwicklungsartig. Vor einer halben Million Jahren sollen unsere Vorfahren zu reden begonnen haben: kommuniziert haben sie, wie andere Lebewesen auch, bereits früher, mit (lockenden, abschreckenden, warnenden) Signalen. Rede erlaubt dem Menschen, sowohl über das Gegenwärtige als auch über das Vergangene und über die Zukunft zu kommunizieren. Dank der Rede hat der Mensch die Grenze der situativen Kommunikation (des Hier und Jetzt) überschritten. Dank der Rede wurde der Mensch zum Kommunikator, zum kommunizierenden Wesen (homo communicans). Als Kommunikationsteilnehmer schuf der Mensch Kultur (homo cultus).

Seit Beginn der Zivilisation sind folgende Etappen zu unterscheiden: Ära der Signale und der Zeichen, Ära des bloßen Sprechens, Ära der Schrift, Ära des Drucks und der Massenkommunikation, Ära der Telekommunikation und der Informatisierung, Ära des Computers (Goban-Klas 2002: 14-17) (auch wenn es nicht auf alle Gemeinschaften beziehbar ist). Medien haben einen bedeutenden Einfluss auf die Entwicklung der menschlichen Kommunikation: heute wird erwartet, dass Informationen schnell, deutlich, genau, immer top-aktuell, auf dem Laufenden (up-to-date) vermittelt werden. Viele Anzeichen der zwischenmenschlichen Kommunikation sind kulturell geprägt, d.h. von Kultur zur Kultur anders. Innerhalb der Kulturtheorie (Oksaar 1988) wird auf das komplexe Zusammenspiel und die wechselseitigen Bedingtheiten zwischen Kultur, Sprache und außersprachlichen Variablen hingewiesen. Daraus lassen sich bestimmte universell menschliche, kulturübergreifende Handlungen, sog. Kultureme beobachten, die typisch für das soziale Zusammenleben innerhalb der einzelnen kulturellen Gruppen sind. Kommunikation ist also kulturgeprägt, eben weil gesellschaftlich, im gesellschaftlichen Kontext verlaufend. Sprache lässt sich nicht auf die reinen verbalen Mittel beschränken, sondern sie wird als ,ein multimodales Ausdruckssystem von verbalen, paraverbalen, extraverbalen und nonverbalen Elementen" verwendet (Bonacchi 2011: 45), daher soll Sprache kulturdeterminiert betrachtet werden. Der Mensch spricht nicht nur mit sprachlichen und nichtsprachlichen Zeichen, sondern mit seinem ganzen Körper, mit seinen Verhaltensweisen, die kulturgeprägt sind (Behavioreme). Die menschliche Kommunikation ist also durch Multimodalität gekennzeichnet (Grucza 2012). Zu unterscheiden sind drei Typen der kommunikativen Einwirkung heute: die monomodale (audio-vokal), bimodale (Sehen und Tasten, Anfassen), multimodale (Hören - Sprechen - Sehen - Tasten). Wenn das verbale Kommunikat durch ein nichtverbales 
Kommunikat des ganzen Körpers (z. B. Gesichtsausdruck) unterstützt wird, ist es lesbarer oder sogar entscheidend (S. und J. Puppel 2008). Die Grundeinheiten des kulturellen Wissens sind (auf der Ebene der Idiokultur und der Polykultur) Kultureme. Es sind Wissensbestände, die aus einer Bedeutungsform und Äußerungsform bestehen; auf ihnen basieren Prozesse der Kulturproduktion und Kulturrezeption (Bonacchi 2011: 59f.). Das kulturemische Wissen konstituiert die Fähigkeit, nicht nur verbale Äußerungen zu produzieren, zu verstehen, zu deuten, sich anzueignen, sondern auch nonverbale Elemente je nach Sinnesmodalität und nach der kognitiven Modalität (Bonacchi 2011: 135), sog. -emische Einheiten: Gesteme, Mimeme, Audeme, Opteme, die die Erzeugung und Deutung von Gesten, Mimik, akustischen Signalen, Tönen, Bildern, visuellen Signalen ermöglichen (Bonacchi 2011: 59). Es sind Wissensbestände über sprachliche und nichtsprachliche Äußerungsformen, die die Prozesse der Kulturproduktion und rezeption, also die Kommunikation, ermöglichen.

\section{Sprachliche und nichtsprachliche Kommunikation}

Die zwischenmenschliche Kommunikation wird von bestimmten Faktoren auf den folgenden vier Ebenen beeinflusst: der verbalen Ebene (Wörter, Sprache), der paraverbalen Ebene (Lautstärke, Stimmlage, Betonung, Hervorheben, Lachen usw.), der nonverbalen Ebene (Körpersprache, also Mimik, Gestik usw.), extraverbalen Ebene (sozialer Kontext, Zeit, Raum, Klima usw.) (Eßer 2010: 172). Die vier Ebenen lassen sich also als sprachliche und nichtsprachliche Einflussfaktoren zusammenfassen.

Seit Jahrhunderten wurden Informationen und wichtige Inhalte den Empfangsmöglichkeiten der Empfänger gerecht gesendet. Symbolische Bilder und Malereien der Urmenschen an den Wänden von Berggrotten, mit der Entdeckung von Schreibgrundlage (Papirus, Papier) war der wesentliche Schritt getan, allerdings war die Schreibkunst nicht sehr allgemein bekannt, daher wurden wesentliche Informationen bildlich verewigt, grafisch vermittelt (Beispiel: die Gnesen-Türen mit Informationen aus dem Leben Christi, sog. Biblia Pauperum). Als das Lesen nicht mehr Rarität war, sondern allen Menschen die zugängliche Normalität, versuchte man, gelesene Inhalte zu verbildlichen, also zu verfilmen; so entstanden visuelle Medien (heute nicht mehr wegzudenken). Das heutige Wahrnehmen, Erkennen geschieht auf der Ebene der Seh-Hörvestehens-Modalität, hauptsächlich: sehen - hören - erkennen. Man behauptet, die heutige Generation sei die Bild-Generation: viele junge Menschen lesen kaum Bücher, statt dessen Comics; Bilder wirken besonders stark auf uns, auf unsere Umwelt, unser Funktionieren, viele Informationen werden durch Bilder geschöpft, durch Bilder lernt man die Umwelt kennen. Nachzudenken ist darüber, ob nicht die heutige 
fortschrittliche Gesellschaft in Richtung der (Rück)Entwicklung zum bloßen Bild-Erkennnen des Urmenschen hinsteuert.

Universelle Handlungen wie Begrüßung, ritualiserte Höflichkeit, Zustimmung, Ablehnung, Ausdruck von Schmerz oder Freude usw. sind in Kulturemen sichtbar, die in den jeweiligen Kulturen jeweils unterschiedlich als Behavioreme realisiert werden (z. B. als Begrüßung Händeschütteln in Deutschland, in Japan Verbeugung, bei Eskimos das Nasenaneinanderreiben). Kultureme sind universell, Behavioreme sind kulturspezifisch, kulturdeterminiert und kulturunterscheidend. Behavioreme sind Einflussfaktoren (innerhalb der Kultureme), also kulturspezifische Ausprägungen der universellen Kultureme; es sind besondere, für verschiedene Kulturen unterschiedliche Verhaltensweisen, die respektiert werden müssen, um am Kommunikationsprozess in einem bestimmten Kulturkontext teilnehmen zu können: verstehen und verstanden werden. $\mathrm{Zu}$ Behavioremen gehören spezifische verbale, nonverbale und paraverbale Formen sowie extraverbale Einflussfaktoren (Eßer 2010: 25). Es sind also sprachliche und nichtsprachliche kulturspezifische Verhaltensweisen, die kommunikationsrelevant sind. Nichtsprachliche Elemente der Kommunikation sind sehr wichtig für das Gelingen der Verständigung, daher sind sie auch glottodidaktisch relevant. Nichtsprachliche (nonverbale) Kommunikation umfasst alle Formen der Kommunikation, in der Bedeutungen, Inhalt und Sinn auch mit unterschiedlichen außersprachlichen Zeichen realisiert werden, solchen, die keine strukturellen Merkmale der natürlichen Sprache aufweisen. Gemeint sind Körpersprache (Gestik, Mimik usw.), Bewegungen (Pantomime), Lautmerkmale (Prosodie, Seufzer), Entfernung (Proxemik) usw.; sie können die Kommunikation unterstützen und ergänzen (oder vernichten) (Kurcz 2005: 218). In Asien wird bspw. Tod nicht als Trauer, sondern als freudiges Ereignis empfunden, dessen äußerer Ausdruck nicht Schwarz, sondern Weiß ist.

Hören - Sehen - Sprechen - Schreiben - Tasten (Anfassen) - Schmecken Riechen, d.h. alle Sinnesmodalitäten werden heute nicht nur bei der face-to-faceKommunikation (ähnlich beim Skypen) verwendet. Menschliche Kommunikation erfolgt auf der Grundlage der simultanen Interaktion von multimodalen körpergestützten (gestischen, mimischen, proxemischen, chronemischen) und gegenstandsgestützten Kommunikationssystemen (Bonacchi 2011: 36, 45-57). Der Sprecher produziert in seiner Interaktion auf der Basis des eigenen Idiolekts sprachliche Äußerungen, die anhand der gemeinsamen polylektalen Schnittmenge an Wissensbeständen (Sprachwissen, Weltwissen, Fachwissen) vom Empfänger verstanden werden können, d.h. um intersubjektiv (also von einer (Menschen) Gruppe akzeptiert oder zumindest respektiert) zu agieren. Die Kommunikationsfähigkeit eines Menschen ist eine dynamische Größe, sie variiert, entwickelt sich (Bonacchi 2011: 102).

Nonverbale Kommunikation umfasst Informationen, die nicht mit Hilfe von Sprache übermittelt werden. Sie ersetzen, erweitern, widersprechen, verändern den 
verbalen Inhalt zur Vermittlung der Aufmerksamkeit, Verstehen und Bewertung, regulieren den Interaktionsablauf, definieren die Beziehungen der Menschen als Kommunikationsteilnehmer zu- und untereinander. Innerhalb der nichtsprachlichen Verhaltensweisen wird Körpersprache genannt (oft als Synonym zur nonverbalen Kommunikation) als eine Komponente des zwischenmenschlichen Verhaltens, die die Interaktion ohne, anstatt, oder zusätzlich zur Sprache bewusst und/oder unbewusst etabliert, aufrechterhält, modelliert und steuert. Körpersprache vermittelt Informationen über alle Kommunikationsteilnehmer, ob Sender oder Empfänger, über ihr Verhältnis zueinander sowie über den Interaktionsgegenstand, sie steuert den Gesprächsverlauf entscheidend mit, durch Blickverhalten, Gestik, Mimik, Haptik, Olfaktorik, Proxemik, Kinesik (Knötig 2010: 228-229). Die sprachliche Kommunikation wird also von unterschiedlichen menschlichen Sinnesmodalitäten begleitet, oder gar entscheidend beeinflusst. Diese Einflüsse sind meistens (großteils) kulturspezifisch und kulturdeterminiert.

Sehr eng mit Sprache sind Gestik und Mimik, die zentralen Elemente der Körpersprache, verbunden: Bewegung der Hände, Arme, Finger (Gestik) sind sprachbegleitend, sie strukturieren, regulieren bzw. ersetzen das Gesagte; manche Gesten, sog. ikonische Zeichen, ersetzen einen ganzen Satz, sind (kulturübergreifend) leicht verständlich, z.B. den Vogel zeigen) (Eßer 2010: 104). Manche Menschen gestikulieren viel, andere weniger, doch auf jeden Fall wird die sprachliche Kommunikation durch Gesten (bzw. durch Mangel an Gesten) wesentlich unterstützt: sie ergänzen und illustrieren die sprachliche Übertragung, sie tragen nicht selten zur Negation des Gesagten bei. Gesten sind einklassige Kodezeichen, einheitliche Ganzheiten, die sich nicht in einfachere Elemente zerlegen lassen; es gibt nur einfache Gesten und/oder Gestenserien. Gesten sind weniger arbiträr als sprachliche Zeichen, können unabhängig von Sprache verstanden werden, meistens sind sie kulturspezifisch zu verstehen (z.B. bejahendes/verneindendes Kopfnicken kann als falscher Freund ausarten). Manche Gesten sind universeller Ausdruck von Gefühlen, Emotionen, Zuständen wie Trauer, Freude, Schmerz (Kultureme, universal). Oftmals reden Frauen expressiver (gestikulierend), was für Gehörlose oder für Menschen mit geringen Sprachkenntnissen kommunikativ behilflich ist (Gebärdensprache). Jeder Mensch hat eigene, für sich typische Gesten. Es gibt Gesten mit spezifischer Bedeutung, die Wörter ersetzen, sog. 'Embleme' (Kurcz 2005: 223) (wie z.B. manche Fingerzeichen, für die Bußgeld vorgesehen ist). Allerdings ist auf kulturelle Unterschiede hinzuweisen: Daumen und Zeigefinger gerundet heißt in den USA: ok; in der Türkei und in Südamerika ist es eine Beleidigung des Gesprächspartners. Zur Mimik gehören menschliche Gesichtsausdrucksmittel, die als Signal individueller Zustände, Anzeichen von Einstellungen, Verhalten verstanden werden; sie modellieren, ersetzen und unterstützen das Gesagte. Sie werden als Kultureme verwendet (als Ausdruck für universelle natürliche Zustände wie Trauer, Freude, 
Schmerz, Angst, Ekel), aber realisiert durch kulturspezifische Behavioreme (Eßer 2010: 214). In manchen Situationen ist Gesichtsausdruck verpönt.

Zur Körpersprache gehört auch das Blickverhalten, also willkürliche oder intentionale Augenbewegungen der Kommunikationsteilnehmer, besonders als Anzeichen der Zuwendung oder Abwendung, Abneigung, je nach Blickdauer (Eßer 2010: 24) (auch das Vermeiden von Blickkontakt, manche Leute schauen ihren Gesprächspartnern nicht in die Augen). Sie sind ebenso kulturell geprägt (kulturunterscheidend, also kulturrelevant). In Skandinavien ist zur Begrüßung kein Körperkontakt geboten: eine unbekannte Person darf man nicht anfassen, nicht küssen, nicht auf die Schultern klopfen; wichtig ist aber der Blickkontakt und leichter Händedruck, ohne die Hand zu schütteln; bei bekannten Frauen ist es erlaubt, kurz auf die Wange zu küssen (ein Mal).

Haptik ist das Aufnehmen von Sinneseindrücken über die Haut, besonders über die Hand (Rischke 2010: 113): (kalte/warme/nasse/zitternde) Hände sind Anzeichen von/geben Informationen (durch das Anfassen, Tasten) über den Gesprächspartner (z. B. Handschlag fast ausschließlich unter (starken) Männern). Bei Papua kitzelte man früher zur Begrüßung den Gesprächspartner am Unterkiefer; bis heute gilt: Bei Unverständnis oder keinem Einverständnis wird geschmatzt.

Proxemik beschreibt das menschliche Raum- und Distanzverhalten, die Entfernung sowohl zu der nichtmenschlichen Umgebung (feste Raumstrukturen: z.B. Wände, halbfixierte: z.B. Möbel), als auch Positionierung des eigenen Körpers im Raum, anderen Menschen gegenüber, oder (un)bewusstes Distanzverhalten (intim bis $45 \mathrm{~cm}$, persönlich bis $120 \mathrm{~cm}$, öffentlich über $360 \mathrm{~cm}$ ). Wichtig ist das Einhalten des persönlichen Raums anderen Personen gegenüber, zwischen den kommunizierenden Personen, Informationen darüber, wie weit/wie nahe darf man jemandem treten. Es gibt hierbei kulturell ziemlich feste Normen (Möglichkeit zu interkulturellen Konflikten) (Knötig 2010: 256-257).

Kinesik umfasst menschliches Bewegungsverhalten (z. B. des Kopfes) im Zusammenspiel mit der Verbalsprache; es lassen sich bedeutungsunterscheidende Bewegungseinheiten sog. 'Kenomorphe' bilden; bedeutend für das Verständnis ist der situative Kontext (sehr kulturgebunden) (Knötig 2010: 152). Kinesik spielt oft mit Proxemik zusammen.

Olfaktorik bedeutet Aufnehmen von Sinneseindrücken über Geruchsorgane, mit Riechen verbunden für sensible Menschen ein ganz wichtiges Zuweisungskennzeichen.

So wie es für jeden Menschen typische sprachliche Ausdrucksweisen gibt, so gibt es auch für jede Person typische Bündel von Elementen nonverbaler Kommunikation, charakteristische nichtsprachliche Merkmale, die Collett (2003) 'tells' nannte: Verhaltens- und Ausdrucksweisen wie Gangart, Bewegungsart, Kleidungsart, sozusagen eine Bewegungscharakteristik, sowie Sprechart, dazu auch andere typische äußere materielle Merkmale wie Gewicht, Körpergröße, Verhaltensweisen können Anzeichen von Schichtenzugehörigkeit sein, also ein Indiz für 
die Stellung innerhalb der Gesellschaft, aber auch Absichten, Einstellungen, Meinungen des Senders ausdrücken. Es sind subjektive Merkmale, die den einzelnen Menschen charakterisieren; durch nonverbale Elemente (z.B. durch Tonfall, prosodische Elemente, Entfernung - Nähe) lassen sich auch Machtverhältnisse (übergeordnet - untergeordnet) ablesen. Sie sind kultutypisch, -abhängig und einzelmenschspezifisch.

Alle die genannten Anzeichen der Körpersprache werden als multisensorische, also auch multimodale Repräsentationen von Wissen durch verschiedene Reizmodi aktiviert, sie werden durch die einzelnen Sinneseindrücke aufgenommen: durch Bewegung der Hände (Gestik), des Gesichts (Mimik), des ganzen Körpers (Kinesik), durch Augenbewegungen (Blickkontakt), durch Raum- und Distanz-Zuweisung (Proxemik), durch Sinneseindrücke über die Haut, besonders auch über Händedruck (Haptik) sowie über Geruchsorgane (Olfaktorik). Einige davon sind dynamisch, mit Bewegung verbunden (Gestik, Mimik, Kinesik, Blickkontakt, Haptik, teilweise auch Proxemik), andere sind statisch begleitende Einflussfaktoren (Olfaktorik, Proxemik), die das Gesagte beeinflussen. Die präferierte Sinnesmodalität bedeutet jeweils die Wahl des Kommunikationskanals, die Bevorzugung einer Sinnesmodalität, des Sehens, Hörens, Tastens, Geschmacks, Geruchs; besonders in Stresssituationen ist es wichtig, die kommunikative Präferenzmodalität des Senders mit der des Empfängers zusammenzukoppeln (S. Puppel, J. Puppel 2008: 1ff).

Letztens entwickelte sich eine neue außersprachliche Ausdrucksform, sog. Emoticon (als Zusammensetzung von Emotion und Ikone, Bild) die Emotionen zum Ausdruck bringen sollen, auch Smileys genannt, vgl. Schneider 2005, 42-46), die mit Hilfe von Computer und Handy entstehen. Sprachliche und nichtsprachliche Kommunikationsmittel des Senders und des Empfängers lassen sich auch in z.B. absichtliche und unabsichtliche/zufällige, korrekt empfangene, hier und jetzt empfangene, nicht empfangene, nicht zeitgemäß gesandte und empfangene Verhaltensweisen gliedern (Littlejohn 1986: 7). Innerhalb der neuen Kulturtechniken entwickelten sich letztens Netiquetten (Bonacchi 2013).

Alle sind kulturdeterminiert, in diesem Sinne kommunikationsrelevant, oftmals -entscheidend. Das Nichtbefolgen bestimmter kultureller Normen führt zum Missverständnis, manchmal auch zum interkulturellen Desaster. Alle sind glottodidaktisch relevant.

\section{Beispiele von Behavioremen}

Behavioreme sind kulturspezifische nichtsprachliche Ausdrucksweisen: zur Begrüßung wird in der Kommunikationsgemeinschaft der Maori in Neuseeland das Nasenaneinanderreiben (Hongi) ausgeführt, diese traditionelle Art gilt bis 
heute (auf Polynesien reibt man zur Begrüßung Nasen aneinander - als Ausdruck von Vertrauen und Bündnis; die Maori glauben, der Schöpfer gab ihnen Leben durch Pusten, Blasen Einflößen der Luft in die Nase; früher begrüßten sich Maori durch Ausstrecken der Zunge, als äußerer Ausdruck der Macht unter Kriegern); in den USA wird beim Händeschütteln zur Begrüßung die Entfernung geschätzt; bei Papua wird jedem die Hand geschüttelt, auch wenn die Menschen gruppiert am Boden sitzen, muss jedem die Hand geschüttelt werden, die Sitzenden fassen den Besucher zusätzlich noch am Bein. Die offene Handfläche vor Händedruck zur Begrüßung zeigt friedliche Absichten unter Kriegern (d. h. kein Messer, keine Waffe in der Hand); aber in Griechenland wird es als Invektive, Beleidigung, Kränkung ausgelegt. In manchen Ländern gilt Begrüßung durch gegenseitige Umarmung mit Kuss auf die Wange auch unter Männern; letztens auch ohne Kuss. Vorsicht ist geboten: Moslems dulden es nicht, von NichtMoslems berührt zu werden, reichen also nicht die Hand (moslemische Frauen umso weniger); das gilt auch in Indien. In Südamerika umarmt man sich zur Begrüßung hauptsächlich unter Männern, begleitet von dem Auf-die-SchulterKlopfen (ohne sich abzuküssen).

In Indien reicht man sich nicht die Hände, um der rituellen Unreinheit vorzubeugen; letztens ist dieses Behaviorem gelockert: in Großstädten reicht man sich die Hände; allerdings muss die wichtigere Person die Hand zuerst reichen). Ehrfurcht (den Eltern, Älteren, dem Guru gegenüber) erweist man durch tiefe Verbeugung vor jemandem und mit seiner rechten Hand die Füße des Älteren anfassen, danach fasst man sich an seinem Herzen und seiner Stirn. Respektlosigkeit jemandem gegenüber erweist man, indem man ihm die Sohle zeigt (die Unterseite des Fußes) oder den Zeigefinger (die rechte Hand ist die saubere Hand, damit isst man; Sohlen der Füße sind schmutzig). Will man jemandem etwas zeigen, etwas erklären, auf etwas hinweisen, macht man das entweder mit voller Handbewegung oder mit dem Unterkiefer. Pünktlichkeit ist in Indien unbekannt, folglich: zum Essen eingeladen soll man diskret (etwa 15-30 Minuten) verspätet kommen; zur Entschuldigung oder aus Scham fasst man sich an seinem Ohr; will man zur Toilette, bewegt man den kleinen Finger der linken Hand.

In ganz Asien und bei Moslems ist es Pflicht, vor dem Betreten des Hauses, Schuhe auszuziehen, zur Begrüßung werden Hände gefaltet (nicht Hand reichen; wenn, dann nur unter Männern). In Japan verbeugt man sich zur Begrüßung und zum Abschied (das ist Pflicht; wer es nicht tut, wird aus der Gruppe ausgeschlossen); bei Verbeugung kein Blickkontakt (Blick ehrfürchtig zu Boden gesenkt); Frauen verbeugen sich mit gefalteten Händen, Männer verbeugen sich mit Händen auf dem Oberschenkel. Je höher das Prestige dem Partner gegenüber, umso tiefer die Verbeugung; bei unbekannten Personen ist eine kurze, neutrale Verbeugung erwartet. Junge Japaner heute wenden sich langsam von 
der Tradition ab: reichen bei Gesprächsrunden die Hand (besonders bei offiziellen Geschäftsgesprächen).

Auch Papst Johannes Paul II. musste eine kulturelle Anpassungsfähigkeit bei offiziellen Anlässen befolgen (nach Latasiewicz 2011): Auf den Fidschi-Inseln musste er die lange Begrüßungsrede mit dem rituellen Ruf Aaa, oj, oj, oj! akzeptieren und danach aus einer Kokosnussschale eine Mixtur trinken. In Togo trank er zur Begrüßung einen Schluck Wasser, den Rest musste er auf den Boden gießen, für die Ahnen; in Ecuador wurde der Papst von den Inkas mit Stille begrüßt (als Ausdruck von Ehrfurcht). Entsprechend kulturdeterminiert waren Geschenke, die er entgegennahm: in Neuseeland bekam er von den Maori als Geschenk einen Mantel aus Federn des Vogels Kiwi, als Ausdruck von Macht. In Bangladesch bekam er einen weißen Turban mit schwarzen Federn - Symbol von Stärke, Ehre und königlicher Macht sowie seidenen Schal und einen kupfernen Gong, als Ausdruck von Erfolg; in asiatischen Ländern wurde er mit Girlanden aus Lotosblumen beschmückt; von der amerikanischen Jugend bekam er Jeans, T-shirt und Hockey-Stock geschenkt; in Madrid bekam er den Mantel eines Torero.

\section{Kommunikation und Fremdsprachenunterricht}

Die Entwicklung der Meinungen zur Kommunikation beeinflussten schon immer auch die Entwicklung des Fremdsprachenunterrichts. Speziell im Hinblick auf den FSU und die Unterrichtsforschung sind die Unterrichtskommunikation und die Unterrichtsinteraktion von Interesse. Es wird untersucht, wie zwischen den Lehrern und Lernern kommuniziert wird (die Unterrichtssprache). Im Vordergrund steht die Frage: Inwiefern ermöglicht die Art der Kommunikation im Unterricht Lernprozesse und Ausdrucksmöglichkeiten, besonders bei den Lernern? Fördert die Unterrichtsinteraktion die Lernprozesse oder werden die Ausdrucksmöglichkeiten der Lerner eher eingeschränkt?

Aus den früheren Modellen der menschlichen Kommunikation, die sich ausschließlich auf sprachliche Kommunikation konzentrierten (als symmetrischer Transmissionsprozess des Inhalts und der Form zwischen dem Sender und dem Empfänger (mit dem (sprachlichen) Kode, dem Kanal (mündlich, schriftlich), dem Kodieren (mit dem synthetischen Kode des Senders) und Dekodieren (mit dem analytischen Kode des Empfängers, L. Zabrocki 1963, 1966a, 1966b 1975)), resultierte der audiolinguale FSU. Diese Unterrichtsmethode entwickelte sich aus der Beobachtung des Erstspracherwerbs durch Kleinkinder; er beruhte prinzipiell auf Zuhören und korrektem (near native) Nachsprechen, um auswendig gelernte korrekte Texte (meistens Dialoge) in Situationen des natürlichen Sprachgebrauchs (ebenso korrekt) anwenden zu können. Auch Unterrichtspro- 
zesse wurden im audiolingualen FSU als symmetrischer Informationsaustausch zwischen Sender (Lehrer/Lerner) und Empfänger (Lerner/Lehrer) aufgefasst. Dabei wurde vorausgesetzt, dass (alle) Lerner den vermittelten Stoff auf die gleiche Art und Weise so aufnehmen, wie ihn der Lehrer präsentiert hat (daher das Auswendiglernen durch Drillübungen-Unterricht). Doch es stellte sich heraus, dass auswendig gelernte Texte noch keine Garantie für situationsadäquaten Sprachgebrauch sind. Kommunikation ist nicht immer effektiv: empfangen wird nicht immer das, was der Sender zu vermitteln beabsichtigte, also was senderseits angestrebt wurde (Gedanken sind zollfrei). Fest steht auch, dass nichtsprachliche Elemente eine besonders wichtige Rolle beim Gelingen oder Misslingen der Kommunikation spielen, da Verständigung multimodal, mit Hilfe von verschiedenen Sinnesmodalitäten verläuft. Das Ziel des Fremdsprachenlernens und -lehrens kann daher Kommunikation als Austausch gesellschaftlicher Relationen sein, sowohl sender- als auch empfängerartige Interpretation verschiedener Zeichensysteme: sprachlicher und nichtsprachlicher (nonverbaler) Zeichen, die alle zusammen einen wesentlichen Beitrag zur Verständigung leisten. Kommunizieren ist also ein intersemiotisches, multimodales, kulturbedingtes Phänomen, bei dem es um kulturdeterminierten Sprachgebrauch geht. Daher eignet sich für den FSU nicht die technizistische Auffassung der Kommunikation als bloßen symmetrischen Transports von Informationen zwischen Sender und Empfänger (wie in der Informationstheorie angenommen wurde). Denn der Alltag des FSU beweist, dass kein Unterrichtsprozess als bloßer symmetrischer Informationsaustausch verläuft; nur selten wird der vom Lehrer intendierte Stoff genau so von den Lernern aufgenommen, denn jeder Lerner lernt anders, je nach seinen individuellen Möglichkeiten und äußeren Lernbedingungen; und außerdem sind nicht alle Lerner gleich aufnahmefähig (Vielau 2003: 239) und am Unterricht interessiert. Deswegen ist für den FSU die Auffassung der Kommunikation als Manifestation gesellschaftlicher Relationen, in denen die Kommunikationsteilnehmer mit Hilfe unterschiedlicher (multimodaler) Zeichensysteme, vor allem aber der kulturdeterminierten Sprachsysteme handeln: versuchen zu verstehen und verstanden zu werden, akzeptabel. Entsprechend ist das Ziel eines jeden Fremdsprachenunterrichts, die Lerner auf effektive Kommunikation vorzubereiten. Das bedeutet, dem Lerner zu verhelfen, sprachliche und nichtsprachliche, kulturdeterminierte Kommunikationsmittel, die in einer Kommunikationsgemeinschaft gelten, intendiert zu verwenden, um bestimmte gesellschaftliche Relationen situationsadäquat realisieren zu können.

Mit der sog. kommunikativen Wende im FSU wurde die Förderung der Kommunikationsfähigkeit der Lerner als kommunikative Kompetenz zum vorrangigen Lernziel des FSU. Der FSU ist eine gesteuerte Art der Kommunikation, sein Ziel ist es, auf möglichst natürliche Kommunikation vorzubereiten. Vor diesem Hintergrund entwickelte sich der kommunikative Ansatz: Kommunikati- 
ve Kompetenz umfasst verbale und nonverbale Kommunikationsmittel sowie die Fähigkeit, sie praktisch, in wirklichen Situationen, im Situationskontext zu verwenden. Auf all das muss der Lerner im FSU vorbereitet werden, damit er sich selbst die Sprache rekonstruieren, erarbeiten, generieren kann (Grucza 2012). Dem können auch sprachliche Schematismen helfen, die im FSU als sprachliche kulturdeterminierte Ausdrucksweisen vermittelt werden (Zenderowska-Korpus 2014).

\section{Homo socius, homo communicans, homo cultus}

Kommunikation beruht auf (natürlichen und konventionellen) Zeichensystemen (Gesten, Töne, Symbole, Pausen, Metaphern, Mehrdeutigkeiten, Unausgesprochenes, Hintergedanken, Wissen und Können). Zwischenmenschliche Kommunikation basiert ebenso auf sprachlichen Mitteln, Worten (akustisch wahrnehmbarer Sprache) wie auch auf nichtsprachlichen Mitteln; Ziel der Kommunikation ist das Erreichen, der Aufbau einer Gemeinschaft und/oder Fortdauer in einer sprachlich-kulturellen Gemeinschaft. Um das Gemeinschaftsziel zu erreichen, muss Verständnis angestrebt werden: sender- und empfängerseits muss Verstand eingesetzt werden, um eine Botschaft zu übermitteln (zu sprechen, zu senden, mitzuteilen, oder zu vertuschen, zu fälschen oder aufzunehmen). Je klarer, transparenter, eindeutiger die Absicht einer Aussage, der Sendung, der Botschaft, umso leichter ist die Empathie des Empfanges, also das Verständnis, die zielgerichtete Kommunikation. Das impliziert aktive Teilnahme am Kommunikationsprozess sowohl des Senders (wer spricht/wer fragt, trägt die Verantwortung für die Antwort des Empfängers) wie auch des Empfängers: es wird aktive, verständnisvolle Teilnahme am Kommunikationsprozess der Gemeinschaftsbildung verlangt. Die Art und Weise der Übermittlung determiniert die Verständigung und die gesamte Kommunikation. Im Kommunikationsprozess wird also kommunikatives Verständnis angestrebt oder (oftmals bewusste) Missverständnisse bzw. Manipulationen. Auf Verständigung müssen sich Kommunikationspartner vorbereiten, einstellen: vom Sender wie vom Empfänger wird aktive Teilnahme erwartet.

Kommunikation lernen kann man nur durch geübtes Kommunizieren. Der Sinn des Fremdsprachenunterrichts ist, das Kommunizieren in der Fremdsprache zu vermitteln und zu lernen. Sprachen lernen kann man nur durch aktives Handeln (Üben) im Hörverstehen, Leseverstehen, Sprechen, Schreiben als integrierte Tätigkeiten, so wie sie im wahren Leben gebraucht werden. Kommunikationsschwierigkeiten offenbaren sich oftmals als Sprachstörungen (z.B. Stottern ist durch Kommunikationsprobleme einer Person verursacht, die durch gezieltes Üben beseitigt werden können). Ein neues Mitglied einer Kommunikationsge- 
meinschaft eignet sich deren Sprache an; ebenso das für diese Gemeinschaft relevante Wertesystem. Das beginnt schon im Kleinkindesalter: Kinder, die ihre erste (Mutter)Sprache erwerben, müssen das phonetische, phonologische, syntaktische, lexikalische System sowie die Semantik erlernen (sich aneignen) - parallel dazu auch noch pragmatisches Können, d.h. die Fähigkeit, die beobachteten und erlernten, generierten Regeln praktisch in konkreten wirklichen Lebenssituationen anzuwenden. Diese pragmatische Fähigkeit ist mit der kognitiven Entwicklung des Kindes verbunden (Piaget 1974). Mit zunehmendem Alter und der fortschreitenden Kenntnis der Muttersprache durch Beobachtung und Interaktion mit seiner Umgebung bekommt (erlangt) das Kind den kommunikativen Schliff, d.h. es lernt, wann, wo, was gesagt werden sollte (Puppel 2001: 168). Je kleiner das Kind, umso natürlicher verläuft die Sprachentwicklung, der Spracherwerb als bloße Anpassung an die Umwelt des Kindes, sich selbst regelnd, sich an die gesellschaftlich-kommunikative Umgebung anpassend. Auch bei Kleinkindern werden Bewusstseinselemente im Spracherwerb unternommen: das Kind merkt sehr wohl, wenn jemand fehlerhaft (zumindest normabweichend) spricht; zweisprachig aufwachsende Kinder merken, in welcher Sprache sie sich an die Mutter, in welcher an den Vater wenden sollen. Je älter der Mensch, umso zielbewusster sein Sprachenlernen. Je älter (erwachsener) der Lerner, umso regelgesteureter sein Sprachlernen.

\section{Zusammenfassung}

Kommunikation ist als gesellschaftlicher Prozess des Bildens, der Übermittlung, und des Interpretierens von Informationen zu verstehen; es ist intendiertes Senden und interpretierendes Empfangen unterschiedlicher (sprachlicher und nichtsprachlicher) Zeichensysteme. Der Mensch als gesellschaftliches Wesen (homo socius) und kommunizierendes Wesen (homo communicans) entwickelte Kultur und wurde zum Kultur-Wesen (homo cultus, im Gegenteil zu homo incultus). Im Fremdsprachenunterricht ist der Bezug zwischen Sprache und Kultur, der sich in sprachlichen und nichtsprachlichen Kommunikationsmitteln äußert, besonders wichtig und glottodidaktisch relevant. Daher sind Sprache und Sprachunterricht als multimodales Kommunikationsgeschehen aufzufassen.

\section{Bibliographie}

Bonacchi, S. 2011. Höflichkeitsausdrücke und anthropozentrische Linguistik. Warszawa: EuroEdukacja.

Bonacchi, S. 2013. (Un)Höflichkeit. Eine kulturologische Analyse Deutsch-Italienisch-Polnisch. Frankfurt am Main/Warszawa: Peter Lang. 
Collett, P. 2003. The book of tells. Toronto: Harper.

DeVito, J.A. 1970. The communication handbook: a dictionary. New York: Harper.

Dobek-Ostrowska, B. 2006. „Miejsce i rola mediów masowych w procesach demokratyzacyjnych". In: Dobek-Ostrowska, B. (Hg.). Media masowe w demokratyzujacych się systemach politycznych: w drodze do wolności stowa i mediów. Wrocław: Wydawnictwo Uniwersytetu Wrocławskiego.

Duranti, A. (Hg.). 2009. A companion to linguistic anthropology. Malden, Mass.: Blackwell.

Eßer, R. 2010. In: Barkowski, H. und H.-J. Krumm. (Hg.). Fachlexikon Deutsch als Fremd- und Zweitsprache. Tübingen: Francke.

Fleischer, M. 2007. Ogólna teoria komunikacji. Wrocław: Wydawnictwo Naukowe Uniwersytetu Wrocławskiego.

Gardner, R.C. 1985. Social psychology and second language learning: the role of attitudes and motivation. London: Edward Arnold.

Goban-Klas, T. 2002. Media i komunikowanie masowe. Warszawa: Wydawnictwo Naukowe PWN.

Grucza, F. 2012. „Zum Gegenstand und zu den Aufgaben der Anthropozentrischen Linguistik, Kulturologie und Kommunikologie sowie zur gegenseitigen Vernetzung dieser Erkenntnisbereiche“. Kwartalnik Neofilologiczny LIX (3). 227-344.

Heath, R.L. und J. Bryant. 2008. Human communication theory and research: concepts, context and challenges. London: Routledge.

Jakobson, R. 1963. Essais de linguistque générale. Paris: Minuit.

Knötig, M. 2010. „Kinesik“. In: Barkowski, H. und H.-J. Krumm. (Hg.). Fachlexikon Deutsch als Fremd- und Zweitsprache. Tübingen: Francke.

Kurcz, I. 2005. Psychologia języka i komunikacji. Warszawa: Scholar.

Lasswell, H.D. 1948. "The structure and the function of communication in society”. In: Bryson, L. (ed.). The communication of ideas. New York: Institute for Religious and Social Studies.

Latasiewicz, M. 2011. Jan Pawet II poza protokołem. Kraków: Rafael.

Lewicka, G. 2007. Glottodydaktyczne aspekty akwizycji języka drugiego a konstruktywistyczna teoria uczenia się. Wrocław: ATUT.

Littlejohn, S.W. 2002. Theories of human communication. Belmont, CA: Wadsworth.

Oksaar, E. 1988. Kulturemtheorie. Ein Beitrag zur Sprachverwendungsforschung. Göttingen: Vandenhoeck and Ruprecht.

Piaget, J. 1974. Biologie und Erkenntnis. Über die Beziehungen zwischen organischen Regulationen und kognitiven Prozessen. Frankfurt: Fischer.

Pisarek, W. 2008. Wstęp do nauki o komunikowaniu. Warszawa: Wydawnictwo Akademickie i Profesjonalne.

Puppel, S. 2001. A conside guide to psycholinguistics. Poznań: Wydawnictwo Poznańskie.

Puppel, S. 2007. „Interlingwalizm czy translingwalizm? Interkomunikacja czy transkomunikacja? Uwagi w kontekście współistnienia języków naturalnych w ramach globalnej wspólnoty kulturowo-językowo-komunikacyjnej”. In: Puppel, S. (red.). Społeczeństwo - kultura - język. W stronę interakcyjnej architektury komunikacji. Poznań: Katedra Ekokomunikacji UAM.

Puppel, J. und S. Puppel. 2008. „Gestosfera jako istotny składnik przestrzeni publicznej”. Oikeios Logos 4. 1-8.

Rieschke, K. 2010. „Haptik“. In: Barkowski, H. und H.-J. Krumm. (Hg.). Fachlexikon Deustch als Fremd- und Zweitsprache. Tübingen: Francke.

Rost-Roth, M. 2010. „Kommunikation“. In: Barkowski, H. und H.-J. Krumm. (Hg.). Fachlexikon Deutsch als Fremd- und Zweitsprache. Tübingen: Francke.

Schneider, S. 2005. „Sprachlernende digital betreuen“. Fremdsprache Deutsch 33. 42-46. 
Shannon, C.E. und W. Weaver. 1949. The mathematical theory of communication. Urbana Chicago: University of Illinois Press.

Skowronek, B. 2008a. „FSU als intersemiotische Kommunikation”. In: Myczko, K., Skowronek, B. und W. Zabrocki (Hg.). Perspektywy glottodydaktyki i językoznawstwa. Tom jubileuszowy z okazji 70. urodzin Profesora Waldemara Pfeiffera. Poznań: Wydawnictwo Naukowe UAM. $107-114$.

Skowronek, B. 2008b. „Fremdsprachenunterricht als Vorbereitung auf die gesellschaftlich effektive Kommunikation“. In: Mikołajczyk, B. und M. Kotin. (Hg.). Terra grammatica. Ideen Methoden-Modelle. Frankfurt am Main: Peter Lang. 393-403.

Skowronek, B. 2013. Glottodidaktik und Fremdsprachenunterricht in der Diskussion. Poznań: Wydawnictwo Naukowe UAM.

Tomaszkiewicz, T. 2006. Przekład audiolingwalny. Warszawa: Wydawnictwo Naukowe PWN.

Vielau, A. 2003. „Die aktuelle Methodendiskussion“. In: Bausch, K.-R., Christ, H. und H.-J. Krumm. (Hg.). Handbuch Fremdsprachenunterricht. Tübingen: Francke.

Watzlavik, P., Beavin, J.H. und D.D. Jackson. 1967/2007. Pragmatics of human communication. New York: W. W. Northon and Company.

Wiener, N. 1954. The human use of human beings. Garden City, N.Y.: Doubleday.

Zabrocki, L. 1963. Wspólnoty komunikatywne w genezie i rozwoju języka niemieckiego. Wrocław: Zakład Narodowy im. Ossolińskich.

Zabrocki, L. 1966a. „Kodematische Grundlagen der Theorie des Fremdsprachenunterrichts“. Glottodidactica 1.3-42.

Zabrocki, L. 1966b. Językoznawcze podstawy metodyki nauczania języków obcych. Warszawa: PWN.

Zabrocki, L. 1975. Kybernetische Modelle der sprachlichen Komunikation. Wrocław/Warszawa/Kraków: Zakład Narodowy im. Ossolińskich.

Zenderowska-Korpus, G. 2004. Sprachliche Schematismen des Deutschen und ihre Vermittlung im Unterricht DaF. Frankfurt am Main: Peter Lang. 